\title{
Is lipopolysaccharide a factor in infectivity of Chlamydia trachomatis?
}

\author{
Sanaa Fadel and Adrian Eley
}

\begin{abstract}
Correspondence
Adrian Eley

a.r.eley@sheffield.ac.uk
\end{abstract}

Received 18 February 2007

Accepted 9 November 2007

\author{
Henry Wellcome Laboratories for Medical Research, Unit of Infection and Immunity, School of \\ Medicine and Biomedical Sciences, University of Sheffield Medical School, Beech Hill Road, \\ Sheffield S10 2RX, UK
}

\begin{abstract}
Lipopolysaccharide (LPS) is a major surface component of Chlamydia trachomatis, as with all Gram-negative bacteria. The effect of C. trachomatis LPS on C. trachomatis infectivity of human epithelial cells was investigated. C. trachomatis LPS and C. trachomatis LPS antibody significantly reduced infectivity, mostly in a dose-dependent manner. As the structure of LPS in C. trachomatis is simple and consists only of lipid A and 3-deoxy-D-manno-octulosonic acid (Kdo), we investigated whether lipid A or Kdo was inhibitory to chlamydial infectivity. Polymyxin B, as a lipid $A$ inhibitor, and Kdo considerably reduced $C$. trachomatis infectivity. With all the LPS inhibitors used, there was greater inhibition against serovar $E$ than serovar LGV. These results suggest a role for LPS in chlamydial infectivity. Elucidation of how LPS acts in infectivity and identification of host-cell receptors would help in understanding pathogenicity.
\end{abstract}

\section{INTRODUCTION}

Several mechanisms by which Chlamydia trachomatis attaches to and infects host cells have been described. Proposed ligands include the major outer-membrane protein (MOMP) (Su et al., 1990; Kuo et al., 1996), heatshock protein 70 (Raulston et al., 1993) and glycosaminoglycans (GAGs) (Zhang \& Stephens, 1992). For the last there is some debate as to whether the GAG is on $C$. trachomatis or is a host molecule (Taraktchoglou et al., 2001; Fadel \& Eley, 2004). However, recent work has shown that interaction of $C$. trachomatis with mammalian cells may be independent of host-cell heparan sulphate GAGs (Stephens et al., 2006). There has also been interest in the entry of $C$. trachomatis into host cells involving cholesterol-rich membrane domains or lipid 'rafts' which appear to be serovar-dependent (Norkin et al., 2001; Stuart et al., 2003). Use of a chlamydial glycolipid exo-antigen vaccine (GLXA; unrelated to lipopolysaccharide) in a vaccine has shown some protection against genital chlamydial infection in a mouse model (WhittumHudson et al., 2001), although in other experimental conditions, it enhances infection (Vora \& Stuart, 2003). However, little evidence has been reported on whether $C$. trachomatis lipopolysaccharide (LPS) might be implicated in infectivity. This is surprising, as LPS is a major surface component of C. trachomatis (Brade, 1999) and has been widely used as the target molecule in laboratory diagnosis by enzyme immunoassay (EIA) (Jones et al., 1984; Chernesky et al., 2001). The present study investigated

Abbreviations: EB, elementary body; GAG, glycosaminoglycan; Kdo, 3-deoxy-D-manno-octulosonic acid; PmB, polymyxin B; TC, tissue culture. whether $C$. trachomatis LPS might be involved in infectivity towards human epithelial cells.

\section{METHODS}

Cell lines and C. trachomatis serovars. The Hec-1B cell line (endometrial carcinoma) was obtained from the American Type Culture Collection (ATCC) and maintained according to the supplier's instructions. Serovar LGV1 was kindly provided by M. Ward (University of Southampton), and C. trachomatis reference strain E/UW-5/CX was a kind gift of D. Dean (Children's Hospital, Oakland Research Institute). Chlamydiae were grown in semiconfluent McCoy cells for 48-72 h in maintenance medium [minimum essential medium Eagle (EMEM) supplemented with cycloheximide $\left.\left(2 \mu \mathrm{g} \mathrm{ml}^{-1}\right)\right]$. The cell suspension containing elementary bodies (EBs) was harvested, and sonicated twice for $10 \mathrm{~s}$ at $15 \mu \mathrm{m}$ amplitude. Cell debris was removed by centrifugation at $500 \mathrm{~g}$ for $15 \mathrm{~min}$, and the remaining suspension was further purified by centrifugation at $30000 \mathrm{~g}$ for $1 \mathrm{~h}$ at $4{ }^{\circ} \mathrm{C}$. The resulting crude $\mathrm{EB}$ pellet was resuspended in $8 \mathrm{ml} \mathrm{PBS}$, sonicated as described above, layered over $30 \%$ urografin (Schering) and centrifuged at $30000 \mathrm{~g}$. Urografin is commonly used to purify EBs in a discontinuous density gradient. The final pellet was resuspended in sucrose phosphate buffer (SPG; $5 \mathrm{mM}$ glutamine, $0.2 \mathrm{M}$ sucrose, $0.2 \mathrm{M}$ phosphate buffer, $\mathrm{pH} 7.4$ ) and stored at $-70{ }^{\circ} \mathrm{C}$ for further use.

Infectivity inhibition assays. Inocula of $C$. trachomatis serovars LGV and $E$ were adjusted so that not more than $\sim 50$ inclusions were present in each field at $\times 400$ magnification $\left[\sim 5 \times 10^{5}\right.$ inclusion forming units (i.f.u.)] in $100 \mu \mathrm{l}$ PBS. The inocula were mixed with dilutions of C. trachomatis LPS, C. trachomatis LPS antibody, polymyxin $B(\mathrm{PmB})$ or synthetic 3-deoxy-D-manno-octulosonic acid (Kdo), and used to infect confluent Hec-1B cell monolayers in 24-well tissue culture (TC) trays, which we have used in previous infectivity studies (Taraktchoglou et al., 2001). C. trachomatis LGV LPS was extracted by the method of Nurminen et al. (1985) and quantified 
using the Limulus amoebocyte lysate (LAL) test (Cambrex Biosciences). Fig. 1 shows the purity of the extracted LPS after electrophoresis through a $14 \%$ acrylamide gel and staining with the Bio-Rad Silver Stain kit. Re mutant LPS from Salmonella enterica 595 (Sigma) was used as a control for C. trachomatis LPS. C. trachomatis LPS $\mathrm{mAb}$ was obtained from Biogenesis and shows activity against all C. trachomatis serovars. Isotype IgG2A (Sigma) was used as a control for C. trachomatis LPS antibody. Both PmB and synthetic Kdo were obtained from Sigma, and PmB nonapeptide (Sigma) and Dglucuronic acid (Sigma) were used as their controls, respectively. The cell monolayers were incubated for $1 \mathrm{~h}$ at $37{ }^{\circ} \mathrm{C}$ in $5 \% \mathrm{CO}_{2}$, and then washed with PBS three times before adding $1 \mathrm{ml}$ EMEM TC fluid supplemented with cycloheximide to each well. After incubation at $37{ }^{\circ} \mathrm{C}$ in $5 \% \mathrm{CO}_{2}$ for $48 \mathrm{~h}$, infected monolayers were fixed, stained, and counted by direct immunofluorescence microscopy using the $C$. trachomatis culture confirmation test (Trinity Biotech). The mean number of inclusion bodies for 25 fields $(\times 400$ magnification $)$ was determined, and compared to the control to calculate the percentage infectivity.

Examination of the binding of LPS to Hec-1B cells using immunohistochemistry. Semiconfluent $\mathrm{Hec}-1 \mathrm{~B}$ cell monolayers in 24-well TC trays were incubated with C. trachomatis LPS for $2 \mathrm{~h}$, washed twice with PBS then incubated with $C$. trachomatis LPS horseradish peroxidase (HRP)-conjugated antibody for $1 \mathrm{~h}$, and the reaction was developed using 4-chloro-1-naphthol. The reaction was viewed with a light microscope at $\times 1000$ magnification. For comparison, the reaction was carried out using Escherichia coli LPS $\mathrm{mAb}$ (Biogenesis). The reaction performed in the absence of $C$. trachomatis LPS was used as a negative control. A positive control included Hec-1B cells infected with serovar LGV1, incubated directly with $C$. trachomatis LPS antibody for $1 \mathrm{~h}$ at room temperature, and developed using the same method.

\section{RESULTS AND DISCUSSION}

\section{Effect of chlamydial LPS on infectivity}

LPS from serovar LGV inhibited the infectivity of $C$. trachomatis in a dose-dependent manner (Fig. 2). At all concentrations of LPS used, there was greater inhibition against serovar E than with serovar LGV, such that at $10 \mu \mathrm{g}$

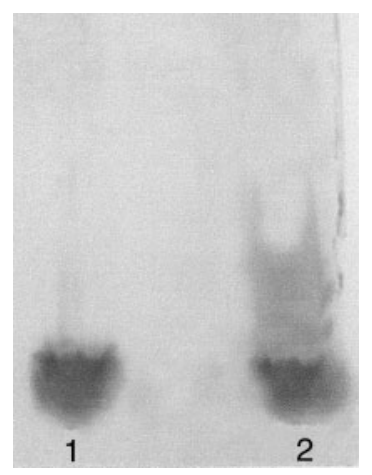

Fig. 1. LPS extracted from C. trachomatis LGV1 (lane 1) and a control E. coli O55:B5 LPS (lane 2) electrophoresed through a $14 \%$ acrylamide gel and stained with silver stain.

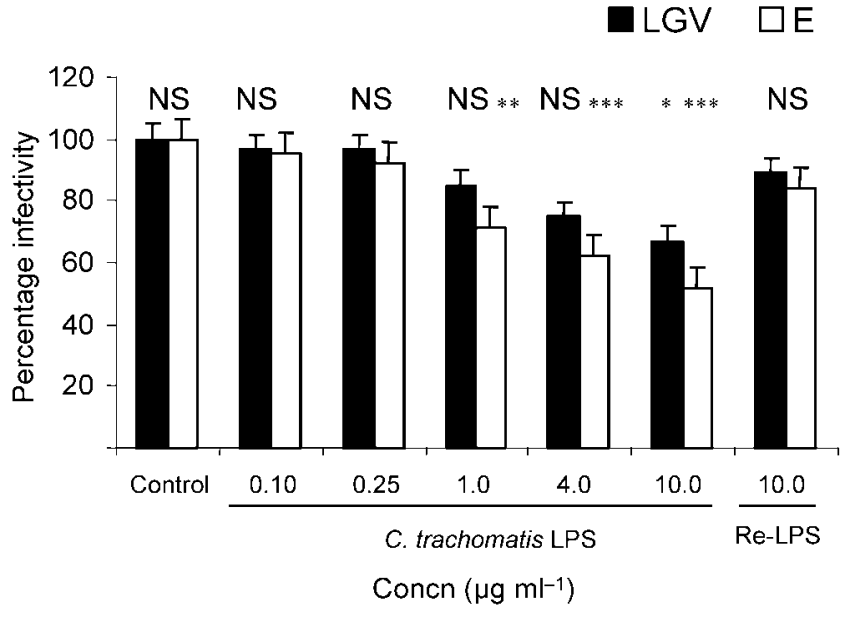

Fig. 2. Infectivity inhibition assay using C. trachomatis LGV LPS. The assay was performed with $C$. trachomatis serovar LGV or serovar $\mathrm{E}$ using Hec-1B cells at $37{ }^{\circ} \mathrm{C}$. Re mutant LPS was used as a control. The figure represents the mean of six experiments, and error bars represent SEM. Results were statistically analysed using one-way-paired ANOVA. ${ }^{*}, P<0.05$; ${ }^{* *}, P<0.01$; ${ }^{* \star *}$, $P<0.001$; NS, non-significant. To compare inhibitory effects on serovars $\mathrm{E}$ and LGV, an unpaired $t$ test was used. Using $10 \mu \mathrm{g}$ $\mathrm{ml}^{-1}$ LPS, the two-tailed $P$ value was $<0.0001$.

$\mathrm{ml}^{-1}$ there was 33 and $48 \%$ inhibition with serovars LGV and $\mathrm{E}$, respectively $(P<0.0001)$. As a control, we used a Re mutant LPS of $S$. enterica, which more closely resembled the overall composition of $C$. trachomatis LPS. Although $S$. enterica LPS is closely related to $C$. trachomatis LPS, the latter has a family-specific epitope (a 2-8 linkage in the core trisaccharide Kdo) which results in a 100-fold decrease in endotoxicity (Kosma, 1999) and is presumably responsible for the difference in infectivity inhibition. It is appreciated that LPS from only one C. trachomatis serovar was tested; however, it is widely believed that the structure of LPS from different serovars is in fact very similar (Heine et al., 2003). C. trachomatis LPS was not toxic to Hec-1B cells at a concentration of $10 \mu \mathrm{g} \mathrm{ml}^{-1}$.

\section{Effect of anti-C. trachomatis antibody on infectivity}

Similar findings were obtained with a commercial $C$. trachomatis LPS antibody (Fig. 3). However, for serovar E at anti-LPS concentrations between 12.5 and $75 \mu \mathrm{g} \mathrm{ml}^{-1}$, there appeared to be more of a plateau than dosedependent effect. At a concentration of $100 \mu \mathrm{g} \mathrm{ml}^{-1}$, the LPS antibody inhibited infectivity of serovars LGV and E by 36 and $54 \%$ respectively. We used an isotype IgG2A antibody as a control. The isotype showed no inhibition of infectivity at the highest antibody concentration used $\left(100 \mu \mathrm{g} \mathrm{ml}^{-1}\right)$, confirming that the inhibitory effect of anti-LPS antibody was not due to a non-specific reaction. 


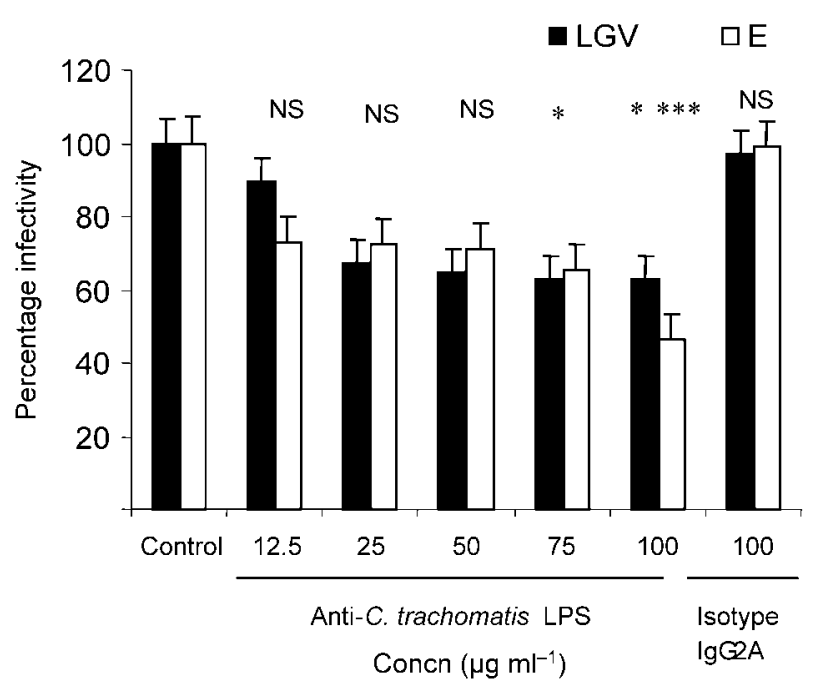

Fig. 3. Infectivity inhibition assay using C. trachomatis LPS antibody. The assay was performed with $C$. trachomatis serovar LGV or serovar $\mathrm{E}$ using $\mathrm{Hec}-1 \mathrm{~B}$ cells at $37{ }^{\circ} \mathrm{C}$. Isotype IgG2A was used as a control. The figure represents the mean of six experiments, and error bars represent SEM. Results were statistically analysed using one-way-paired ANOVA. *, $P<0.05$, **, $P<0.01 ;{ }^{* *}, P<0.001$; NS, non-significant.

\section{Effect of PmB and Kdo on infectivity}

$\mathrm{PmB}$ is often used as an LPS inhibitor as it specifically binds to lipid A (Morrison \& Jacobs, 1976). However, as it also has some antibacterial activity, its use in infectivity experiments could be misleading. Nevertheless, as the drug acts almost exclusively on extracellular pathogens, an antibiotic effect on C. trachomatis, which is obligately intracellular, seems unlikely (Redecke et al., 1998). Moreover, we used $\mathrm{PmB}$ nonapeptide as a control. This cationic cyclic peptide is derived from PmB. Although it has extremely low antimicrobial activity, it is still capable, like PmB, of binding to LPS (Tsubery et al., 2002). At $100 \mu \mathrm{g} \mathrm{ml}{ }^{-1}, \mathrm{PmB}$ showed inhibition of infectivity of serovars LGV and E by 49 and $65 \%$, respectively (Fig. 4), which again is suggestive of LPS, especially lipid A, involvement. Similarly, $\mathrm{PmB}$ nonapeptide showed a comparable inhibitory effect, confirming that inhibition by $\mathrm{PmB}$ was due to LPS binding, and not due to antibacterial activity. As the structure of LPS in C. trachomatis is simple and consists only of lipid A and Kdo, we next investigated whether Kdo was inhibitory. Unfortunately, Kdo from C. trachomatis was unavailable, so instead we used synthetic Kdo. Interestingly, at $100 \mu \mathrm{g}$ $\mathrm{ml}^{-1} \mathrm{Kdo}$, there was inhibition of serovars LGV and $\mathrm{E}$ by 39 and $57 \%$, respectively (Fig. 5). As a control, we used another synthetic carbohydrate, D-glucuronic acid, which like Kdo is negatively charged and is also found in chlamydial LPS; this showed no inhibitory effect and confirmed the specificity of Kdo.

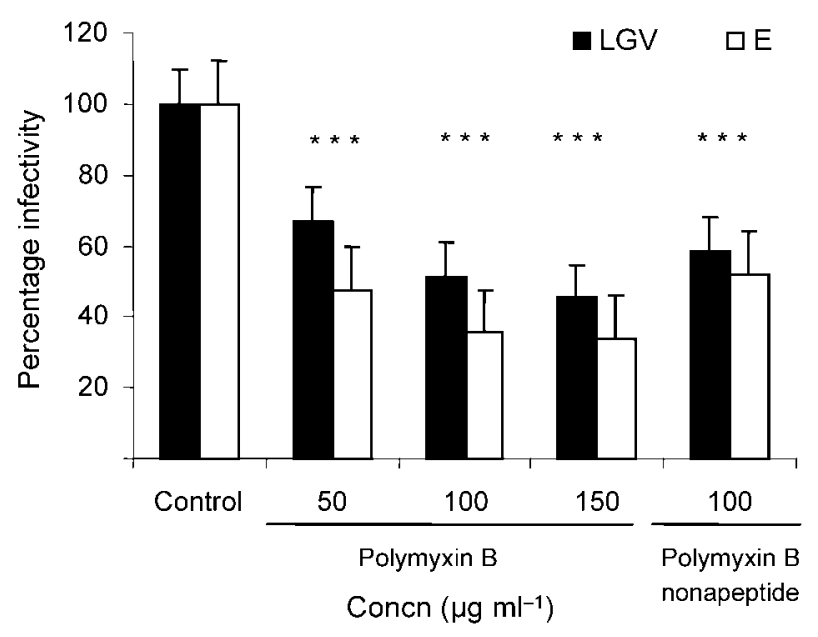

Fig. 4. Infectivity inhibition assay using $\mathrm{PmB}$. The assay was performed with $C$. trachomatis serovar LGV or serovar $E$ using Hec-1B cells at $37{ }^{\circ} \mathrm{C}$. PmB nonapeptide was used as a control. The figure represents the mean of three experiments, and error bars represent SEM. Results were statistically analysed using oneway-paired ANOVA. * $P<0.05$; ${ }^{\star *}, P<0.01$; ${ }^{\star \star *}, P<0.001$; NS, non-significant.

\section{Immunohistochemistry}

Another approach to investigate the role of LPS in chlamydial adherence was by immunohistochemistry using a specific C. trachomatis LPS HRP-conjugated antibody. As shown in Fig. 6, incubation of Hec-1B cells with chlamydial LPS followed by $C$. trachomatis LPS antibody showed a

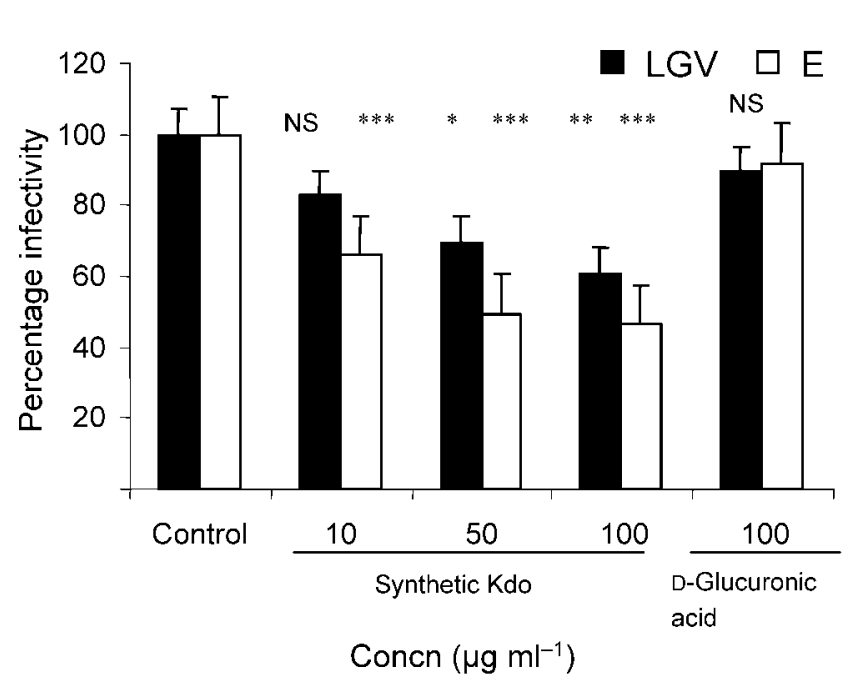

Fig. 5. Infectivity inhibition assay using synthetic Kdo. The assay was performed with C. trachomatis serovar LGV or serovar E using Hec1B cells at $37{ }^{\circ} \mathrm{C}$. D-Glucuronic was used as a control. The figure represents the mean of three experiments, and error bars represent SEM. Results were statistically analysed using one-way-paired ANOVA. ${ }^{*}, P<0.05 ;{ }^{\star \star}, P<0.01$; ${ }^{\star \star \star}, P<0.001$; NS, non-significant. 

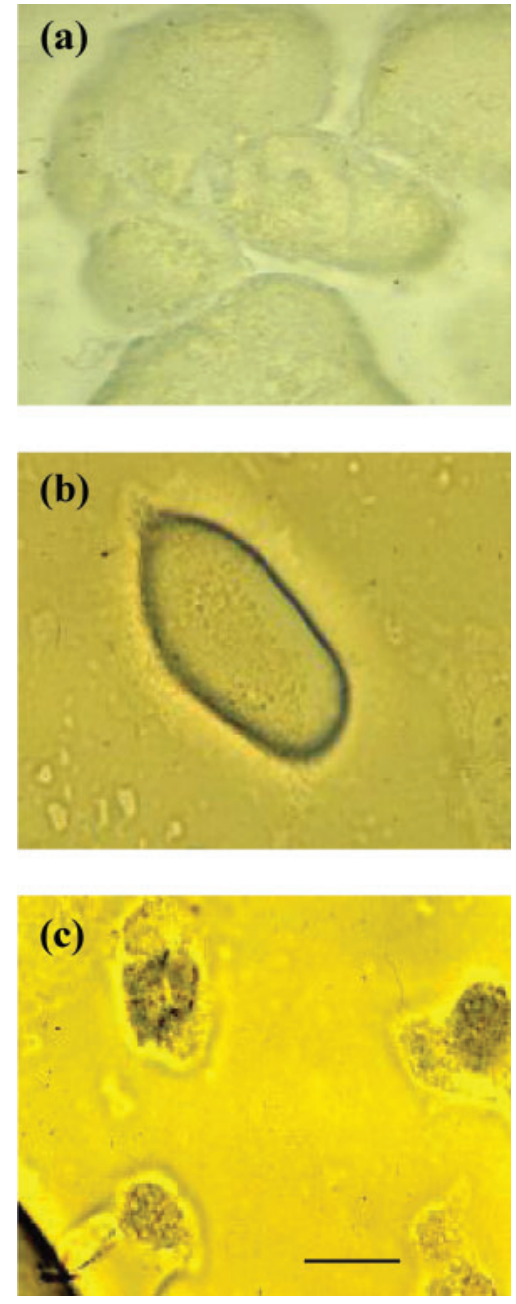

Fig. 6. Examination of the binding of $C$. trachomatis LPS to Hec1B cells using immunohistochemistry. Cells were incubated with chlamydial LPS for $2 \mathrm{~h}$, washed, then incubated with $C$. trachomatis HRP-conjugated antibody for $1 \mathrm{~h}$, and the reaction was developed and immediately viewed with a light microscope $(\times 1000)$. (a) Negative control in which C. trachomatis LPS was omitted. (b) Hec-1B cells incubated with C. trachomatis LPS, followed by $C$. trachomatis antibody. (c) Positive control, in which Hec-1B cells infected with serovar LGV1 were incubated with $C$. trachomatis antibody. Bar, $10 \mu \mathrm{m}$.

positive reaction in the form of a deep-purple colour distributed evenly at the edges of the cells. Hec-1B cells infected with LGV1 serovar and then incubated with $C$. trachomatis LPS antibody showed that most of the cells displayed dark staining. This was used as a positive control. The reaction carried out in the absence of LPS was negative.

In this study, all the findings suggest that $C$. trachomatis LPS plays a role in infectivity towards epithelial cells, although some of our data support the idea that infectivity is complex and likely involves more than one moiety. That
C. trachomatis LPS might be playing a role in infectivity was demonstrated using LPS, C. trachomatis LPS antibody, $\mathrm{PmB}$ and Kdo as inhibitors. To the best of our knowledge, this is the first report of experiments with PmB nonapeptide and D-glucuronic acid. Immunohistochemistry experiments also suggested that $C$. trachomatis LPS binds to Hec-1B cells. However, the nature of the receptor to which LPS binds remains unclear. An approach using heparin affinity chromatography showed that LPS did not bind to heparin, indicating that the host receptor is unlikely to belong to the GAGs (data not shown). Nevertheless, different results have been shown by other workers ( $\mathrm{Su}$ et al., 1990; Byrne et al., 1993) in C. trachomatis LPS antibody experiments, and although their C. trachomatis LPS antibody showed little inhibitory effect, there were several key differences in methodology. The serovar tested was B, the cells used were HaK (Syrian hamster kidney), and the inoculum size was significantly larger. All these differences make it extremely difficult to compare findings. We also realize that the Kdo we used in our experiments was synthetic and that inhibition by Kdo could have been due, at least in part, to its negative charge; however, these results do suggest that further work should be initiated.

We should also make it clear that even though we used a standard method for LPS quantification, it is known that C. trachomatis LPS has very low endotoxic activity (Kosma, 1999), and therefore the actual amounts of LPS used in our experiments are likely to have been higher than those measured.

Also of note in our experiments was the fact that unlike many other inhibition studies with $C$. trachomatis, especially those using GAG inhibitors, in which serovar LGV is almost always inhibited to a greater degree than serovar E (Taraktchoglou et al., 2001; Fadel \& Eley, 2004), the converse was true when LPS was the target for infectivity. It may suggest that this possible target of infectivity is more important for serovar E than serovar LGV.

LPS, or in some cases lipooligosaccharide (LOS), has been reported to be important in bacterial adherence (Jacques, 1996), and more specifically in attachment of several bacterial pathogens of the genital and respiratory tracts, including Neisseria gonorrhoeae (Harvey et al., 2000), Haemophilus ducreyi (Alfa \& DeGagne, 1997) and Actinobacillus pleuropneumoniae (Belanger et al., 1990). For the last organism, further evidence shows that its adherence to porcine tracheal cells is inhibited by $A$. pleuropneumoniae LPS antibody (Paradis et al., 1999). The same workers have shown that the active component of its LPS in adherence is more likely to be Kdo than lipid A (Paradis et al., 1994). Our data also show that Kdo from $C$. trachomatis LPS may play a significant role in $C$. trachomatis infectivity that is therefore not unique.

Although it is premature to consider if lipid 'rafts' might be related in any way to the role of LPS in infectivity, recent evidence suggests that A. pleuropneumoniae LPS binds to the phospholipid phosphatidylethanolamine (PE) of the 
host (Jeannotte et al., 2003). This is of interest, as back in 1991, C. trachomatis was found to bind PE as well as asialoGM1 and asialo-GM2, and at that time these molecules were considered to be putative host-cell receptors (Krivan et al., 1991).

Several studies have reported that chlamydial LPS is present on the surface of infected cells (Campbell et al., 1994; Wyrick et al., 1994), albeit in low quantities; therefore, it may be that C. trachomatis, like A. pleuropneumoniae, uses LPS as an adhesin. However, whether surface exposure of LPS is greater on chlamydial reticulate bodies (RBs) than on EBs (Collett et al., 1989) remains an important question in determining whether LPS is more likely to be implicated in infectivity mechanisms other than adherence. Further work is therefore warranted to investigate the role of $C$. trachomatis LPS in infectivity, including the use of chlamydial lipid A and Kdo, despite limitations caused by difficulties in their extraction.

\section{REFERENCES}

Alfa, M. J. \& DeGagne, P. (1997). Attachment of Haemophilus ducreyi to human foreskin fibroblasts involves LOS and fibronectin. Microb Pathog 22, 39-46.

Belanger, M., Dubreuil, D., Harel, J., Girard, C. \& Jacques, M. (1990). Role of lipopolysaccharide in adherence of Actinobacillus pleuropneumoniae to porcine tracheal rings. Infect Immun $\mathbf{5 8}$, 3523-3530.

Brade, H. (1999). Chlamydial lipopolysaccharide. In Endotoxin in Health and Disease, pp. 229-242. Edited by H. Brade, S. M. Opal, S. N. Vogel \& D. C. Morrison. New York: Marcel Dekker.

Byrne, G. I., Stephens, R. S., Ada, G., Caldwell, H. D., Su, H., Morrison, R. P., Van der Pol, B., Bavoil, P. \& other authors (1993). Workshop on in vitro neutralization of Chlamydia trachomatis: summary of proceedings. J Infect Dis 168, 415-420.

Campbell, S., Richmond, S. J., Yates, P. S. \& Storey, C. C. (1994). Lipopolysaccharide in cells infected by Chlamydia trachomatis. Microbiology 140, 1995-2002.

Chernesky, M., Jang, D., Copes, D., Patel, J., Petrich, A., Biers, K., Sproston, A. \& Kapala, J. (2001). Comparison of a polymer conjugate-enhanced enzyme immunoassay to ligase chain reaction for diagnosis of Chlamydia trachomatis in endocervical swabs. J Clin Microbiol 39, 2306-2307.

Collett, B. A., Newhall, W. J., Jersild, R. A., Jr \& Jones, R. B. (1989). Detection of surface-exposed epitopes on Chlamydia trachomatis by immune electron microscopy. J Gen Microbiol 135, 85-94.

Fadel, S. \& Eley, A. (2004). Chlorate: a reversible inhibitor of proteoglycan sulphation in Chlamydia trachomatis-infected cells. J Med Microbiol 53, 93-95.

Harvey, H. A., Porat, N., Campbell, C. A., Jennings, M., Gibson, B. W., Phillips, N. J., Apicella, M. A. \& Blake, M. S. (2000). Gonococcal lipooligosaccharide is a ligand for the asialoglycoprotein receptor on human sperm. Mol Microbiol 36, 1059-1070.

Heine, H., Muller-Loennies, S., Brade, L., Lindner, B. \& Brade, H. (2003). Endotoxic activity and chemical structure of lipopolysaccharides from Chlamydia trachomatis serotypes $\mathrm{E}$ and L2 and Chlamydophila psittaci 6BC. Eur J Biochem 270, 440-450.

Jacques, M. (1996). Role of lipo-oligosaccharides and lipopolysaccharides in bacterial adherence. Trends Microbiol 4, 408-409.
Jeannotte, M. E., Abul-Milh, M., Dubreuil, J. D. \& Jacques, M. (2003). Binding of Actinobacillus pleuropneumoniae to phosphatidylethanolamine. Infect Immun 71, 4657-4663.

Jones, M. F., Smith, T. F., Houglum, A. J. \& Herrmann, J. E. (1984). Detection of Chlamydia trachomatis in genital specimens by the Chlamydiazyme test. J Clin Microbiol 20, 465-467.

Kosma, P. (1999). Chlamydial lipopolysaccharide. Biochim Biophys Acta 1455, 387-402.

Krivan, H. C., Nilsson, B., Lingwood, C. A. \& Ryu, H. (1991). Chlamydia trachomatis and Chlamydia pneumoniae bind specifically to phosphatidylethanolamine in HeLa cells and to GalNAc beta 14Gal beta 1-4GLC sequences-found in asialo-GM1 and asial-GM2. Biochem Biophys Res Commun 175, 1082-1089.

Kuo, C., Takahashi, N., Swanson, A. F., Ozeki, Y. \& Hakomori, S. (1996). An $\mathrm{N}$-linked high-mannose type oligosaccharide, expressed at the major outer membrane protein of Chlamydia trachomatis, mediates attachment and infectivity of the microorganism to HeLa cells. J Clin Invest 98, 2813-2818.

Morrison, D. C. \& Jacobs, D. M. (1976). Binding of polymyxin B to the lipid A portion of bacterial lipopolysaccharides. Immunochemistry 13, 813-818.

Norkin, L. C., Wolfson, S. A. \& Stuart, E. S. (2001). Association of caveoli with Chlamydia trachomatis inclusions at early and late stages of infection. Exp Cell Res 266, 229-238.

Nurminen, M., Rietschel, E. T. \& Brade, H. (1985). Chemical characterization of Chlamydia trachomatis lipopolysaccharide. Infect Immun 48, 573-575.

Paradis, S. E., Dubreuil, D., Rioux, S., Gottschalk, M. \& Jacques, M. (1994). High-molecular-mass lipopolysaccharides are involved in Actinobacillus pleuropneumoniae adherence to porcine respiratory tract cells. Infect Immun 62, 3311-3319.

Paradis, S. E., Dubreuil, J. D., Gottschalk, M., Archambault, M. \& Jacques, M. (1999). Inhibition of adherence of Actinobacillus pleuropneumoniae to porcine respiratory tract cells by monoclonal antibodies directed against LPS and partial characterization of the LPS receptors. Curr Microbiol 39, 313-320.

Raulston, J. E., Davis, C. H., Schmiel, D. H., Morgan, M. W. \& Wyrick, P. B. (1993). Molecular characterization and outer membrane association of a Chlamydia trachomatis protein related to the hsp70 family of proteins. J Biol Chem 268, 23139-23147.

Redecke, V., Dalhoff, K., Bohnet, S., Braun, J. \& Maass, M. (1998). Interaction of Chlamydia pneumoniae and human alveolar macrophages: infection and inflammatory response. Am J Respir Cell Mol Biol 19, 721-727.

Stephens, R. S., Poteralski, J. M. \& Olinger, L. (2006). Interaction of Chlamydia trachomatis with mammalian cells is independent of host cell surface heparan sulphate glycosaminoglycans. Infect Immun 74, 1795-1799.

Stuart, E. S., Webley, W. C. \& Norkin, L. C. (2003). Lipid rafts, caveolae, caveolin-1, and entry by Chlamydiae into host cells. Exp Cell Res 287, 67-78.

Su, H., Watkins, N. G., Zhang, Y. X. \& Caldwell, H. D. (1990). Chlamydia trachomatis-host cell interactions: role of the chlamydial major outer membrane protein as an adhesin. Infect Immun 58, 1017-1025.

Taraktchoglou, M., Pacey, A. A., Turnbull, J. E. \& Eley, A. (2001). Infectivity of Chlamydia trachomatis serovar LGV but not E is dependent on host cell heparan sulphate. Infect Immun 69, 968-976.

Tsubery, H., Ofek, I., Cohen, S., Eisenstein, M. \& Fridkin, M. (2002). Modulation of the hydrophobic domain of polymyxin B nonapeptide: 
effect on outer-membrane permeabilization and lipopolysaccharide neutralization. Mol Pharmacol 62, 1036-1042.

Vora, G. J. \& Stuart, E. S. (2003). A role for the glycolipid exoantigen (GLXA) in chlamydial infectivity. Curr Microbiol 46, 217-223.

Whittum-Hudson, J. A., Rudy, D., Gerard, H., Vora, G., Davis, E., Haller, P. K., Prattis, S. M., Hudson, A. P., Saltzman, W. M. \& Stuart, E. S. (2001). The anti-idiotypic antibody to chlamydial glycolipid exoantigen (GLXA) protects mice against genital infection with a human biovar of Chlamydia trachomatis. Vaccine 19, 4061-4071.

Wyrick, P. B., Choong, J., Knight, S. T., Goyeau, D., Stuart, E. S. \& McDonald, A. B. (1994). Chlamydia trachomatis antigens on the surface of infected human endometrial epithelial cells. Immunol Infect Dis 4, 131-141.

Zhang, J. P. \& Stephens, R. S. (1992). Mechanism of Chlamydia trachomatis attachment to eukaryotic host cells. Cell 69, 861-869. 